\title{
RANKING DE INNOVACIÓN PERIODISTICA 2014 EN ESPAÑA. SELECCIÓN Y ANÁLISIS DE 25 INICIATIVAS
}

\section{Ranking of journalistic innovation in Spain. Analysis and classification of 25 initiatives}

\author{
Alicia De-Lara-González, Félix Árias-Robles, Miguel Carvajal-Prieto y José- \\ Alberto García-Avilés
}
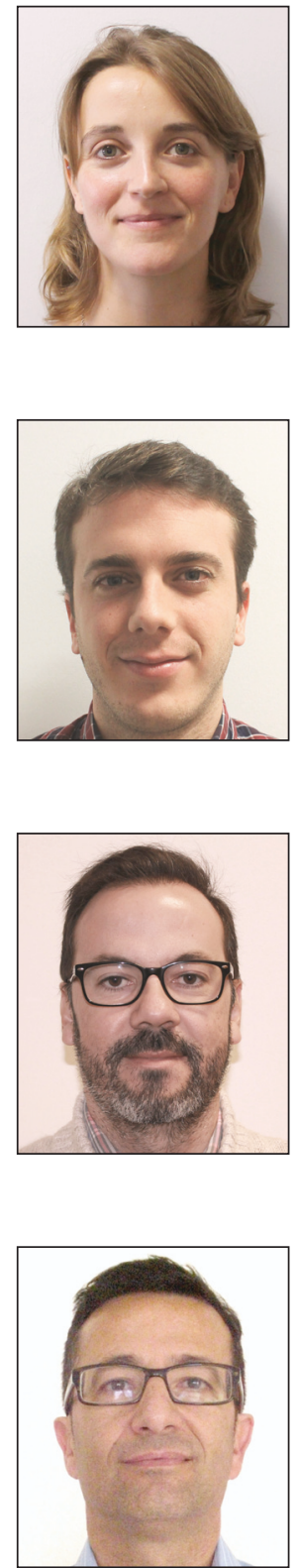

Alicia De-Lara-González es profesora ayudante doctor en la Universidad Miguel Hernández de Elche (UMH). Dirige el Grupo de Investigación de la Comunicación en la Comunidad Valenciana (Gicov). Licenciada en publicidad y relaciones públicas por la Universidad de Alicante y en periodismo por la Universidad de Murcia. Ha escrito artículos y participado en congresos sobre producción periodística, publicidad y tratamiento informativo. Ha disfrutado de dos estancias de investigación en universidades anglosajonas y es redactora jefe de la revista de divulgación científica UMH sapiens.

http://orcid.org/0000-0001-5556-6317

a.lara@umh.es

Félix Árias-Robles, investigador en formación en la Universidad Miguel Hernández de Elche (UMH), es licenciado en periodismo por la UMH y Máster en investigación en periodismo por la Universidad Complutense. Coordina un módulo sobre nuevas narrativas en el Máster en innovación en periodismo de la $U M H$ y realiza su tesis doctoral sobre la influencia de la hipertextualidad en la producción y la recepción de los contenidos periodísticos.

http://orcid.org/0000-0001-5910-1541

flxarias@gmail.com

Miguel Carvajal-Prieto es profesor contratado doctor en la Universidad Miguel Hernández de Elche $(U M H)$. Es licenciado en periodismo y doctor en empresa informativa por la Universidad de Navarra. Dirige el Máster en innovación en periodismo de la UMH, ha sido visiting scholar en el Donald Mc Gannon Center de la Fordham University (Nueva York) y profesor en la Universidad Católica San Antonio. Su investigación se centra en el área de economía, estrategia e innovación en periodismo.

http://orcid.org/0000-0001-6547-6171

mcarvajal@umh.es

José-Alberto García-Avilés es profesor titular de periodismo en la Universidad Miguel Hernández de Elche (UMH). Es Bachelor of Arts por la National University of Ireland, licenciado en periodismo y doctor en comunicación por la Universidad de Navarra. Trabajó en varios canales de TV, en la productora Euroview y en la Facultad de Comunicación de la Universidad de Navarra. Imparte docencia en el Master de innovación en periodismo de la UMH. Sus temas de investigación son la innovación en periodismo, las prácticas informativas y la calidad de los contenidos.

http://orcid.org/0000-0001-7854-3476

jose.garciaa@umh.es

Universidad Miguel Hernández de Elche Facultad Ciencias Sociales y Jurídicas, Departamento de Ciencias Sociales y Humanas Edif. Torreblanca. Avda de la Universidad, s/n. 03202 Elche, Alicante, España

Artículo recibido el 09-01-2015

Aceptación definitiva: 23-02-2015 


\title{
Resumen
}

El Ranking de innovación periodística 2014 selecciona y analiza las 25 iniciativas innovadoras que conforman el motor del cambio en la industria informativa española. Esta clasificación combina la consulta a expertos con el uso de herramientas de medición. Se elaboró una base de datos compuesta por 196 iniciativas que se estudiaron en función del grado, el objeto y la naturaleza de cada innovación. El marco temporal del estudio abarcó desde julio de 2013 hasta julio de 2014. Los resultados apuntan que la innovación periodística en España se desarrolla en los márgenes de la industria informativa tradicional y pone en valor proyectos periodísticos no convencionales que en algunos casos resultan poco conocidos aún entre los usuarios.

\section{Palabras clave}

Innovación; Periodismo; Ranking; Medios de comunicación; Medios online.

\begin{abstract}
The 2014 Ranking of Journalism Innovation systematically analyses and classifies the innovative ideas that have become the driving force of change in the Spanish news industry. The ranking is based on a detailed analytical matrix, which classifies a total of 196 initiatives from various journalistic areas using a methodology that combines the input of experts with the use of measurement tools and metrics. The study ranges from July 2013 to July 2014 . The results show that journalistic innovation is being implemented at the margins of the traditional Spanish media industry and highlight alternative projects which in some cases are not yet very well known among the public.
\end{abstract}

\section{Keywords}

Innovation; Journalism; Ranking; News media; Online media.

De-Lara-González, Alicia; Árias-Robles, Félix; Carvajal-Prieto, Miguel; García-Avilés, José-Alberto (2015)."Ranking de innovación periodística 2014 en España. Selección y análisis de 25 iniciativas”. El profesional de la información, v. 24, n. 3, pp. 235-245

\section{Introducción: la innovación en periodismo}

En los últimos años, el concepto de innovación se ha convertido en lugar común en diversos sectores de la actividad empresarial, tecnológica y social (Ramírez-Alujas, 2011), aunque el término casi ha llegado a verse desprovisto de su sentido por usarse demasiado (Fagerberg, 2004). La innovación implica la capacidad de asumir los cambios y usar habilidades creativas para detectar un problema, encontrar una solución original y desarrollarla exitosamente (EscorsaCastells; Valls-Pasola, 2003). Consiste en implementar un nuevo concepto, producto o servicio en un mercado concreto de forma disruptiva, es decir, que esa novedad altere el modo tradicional en el que se venían realizando las cosas (Christensen, 1997).

Este campo académico ha despertado el interés de los investigadores desde múltiples perspectivas:

- la gestión de los medios (Dal-Zotto; Van-Kranenburg, 2008; Baumann, 2013);

- las nuevas tecnologías y servicios (Dogruel, 2013);

- las relaciones con la "audiencia social" (Bruns, 2014);

- los modelos de negocio emergentes (Carvajal; GarcíaAvilés; González, 2012).

Según se aprecia en los medios que han introducido estrategias innovadoras, la innovación conlleva la renovación de los procesos productivos (Westlund; Krumsvik, 2014) integrando las personas y los recursos al servicio de tales procesos, y no al revés (García-Avilés, 2012).
En el periodismo, la innovación se lleva a cabo mediante "mutaciones" en ámbitos relacionados con la tecnología, la comunicación y la organización, según Boczkowski (2004, p. 11). A partir del estudio de Boczkowski, Steensen (2009) subraya que la cultura de trabajo, la gestión de los directivos, el uso adecuado de la tecnología y la iniciativa de algunos periodistas también inciden en el grado de innovación. Ello supone centrarse no sólo en los productos, sino también en los procesos que crean dichos productos y que pueden incorporar innovaciones de modo tangible o intangible (Dogruel, 2014, pp. 57-8). La autonomía de las redacciones online frente a las del medio matriz permite innovar en la producción informativa, en la organización del trabajo, en los formatos narrativos o en las aplicaciones tecnológicas (Micó; Masip; Domingo, 2013).

Diversos estudios de caso ilustran los procesos de innovación en periodismo. El trabajo de García-Avilés, Kaltenbrunner y Meier (2014) revela que las prácticas de la cultura impresa ralentizaron la implantación de las redacciones integradas en varios medios europeos. Weiss y Domingo (2010) consideran las redacciones online como comunidades de práctica profesionales, donde los periodistas tratan de vencer resistencias y difundir las innovaciones. Otros estudios apuntan que las nuevas tecnologías permiten implementar productos, servicios o procesos innovadores que satisfacen necesidades de los usuarios (Nee, 2013).

El periodismo online se encuentra en plena ebullición, con el surgimiento de iniciativas que buscan la rentabilidad me- 
diante propuestas a priori innovadoras (Bruno; Nielsen, 2012), mientras que los medios consolidados llevan a cabo estrategias para afrontar los cambios disruptivos en la industria, como indica el Innovation report de The New York times (2014, p. 1). Numerosos medios locales tratan de innovar para afrontar retos creativos y económicos en entornos competitivos (Van-Kerkhoven; Bakker, 2014).

En España, el periodismo digital muestra una evolución progresiva desde mediados de los noventa, desde la adaptación de contenidos de los medios tradicionales, hasta el uso de formatos específicos y de nuevas prácticas profesionales propias (López-García, 2004; Salaverría, 2005). Surgieron innovaciones técnicas y conceptuales, tales como el uso de los multimedia, la interactividad y el hipertexto, plasmando un modelo de periodismo que buscaba la interacción con la audiencia (Larrondo-Ureta, 2005). La mayoría de los grupos multimedia españoles implantaron estrategias de convergencia, con diversos niveles de producción crossmedia o integración de redacciones (López-García; PereiraFariña, 2010). La convergencia a menudo se tradujo en un incremento de la carga laboral de los profesionales, una mayor polivalencia con el fin de reducir costes (Noguera-Vivo, 2010). El vídeo cobró importancia en un proceso de mayor multimedialidad y audiovisualización de la información online (Guallar; Rovira; Ruiz, 2010).

Como pone de manifiesto el Informe anual de la profesión periodística (APM, 2014), en España desde 2008 han surgido más de 450 nuevos medios, la mayoría proyectos informativos digitales promovidos por periodistas. Los cibermedios nativos hiperlocales conceden especial protagonismo a los ciudadanos y ofrecen novedosas fórmulas de contenidos generados por los usuarios (García-de-Torres, 2010). También surgen proyectos con financiación mediante crowdfunding (Casero-Ripollés, 2010). El éxito de la financiación de estos proyectos se halla directamente vinculado a su capacidad para movilizar comunidades de usuarios, que en general son pequeñas (Fumero-Reverón, 2011). Al mismo tiempo, como apunta Gumersindo Lafuente (cit. en Ponce-Aguirre, 2013), es necesario que los nuevos medios profundicen de forma más sistemática en la experimentación, renovando productos y lenguajes, donde la innovación tecnológica esté al servicio de una audiencia crítica y participativa.

\section{Metodología}

El Ranking de innovación periodística responde al interés por establecer unos parámetros homogéneos que ayuden a identificar los aspectos innovadores del periodismo contemporáneo español ${ }^{1}$. Para llevar a cabo un trabajo así, es necesario posicionarse sobre tres puntos clave (Bleyen et al., 2014):

a) concepto de innovación; b) objeto de la innovación;

c) grado de la innovación.

La ausencia de consenso académico y la variedad de enfoques aconsejaba a priori no emprender un ranking de estas características, pero se alcanzó un punto de partida robusto sobre esas tres premisas.

\section{La innovación periodística en España tie- ne lugar en los márgenes de la industria informativa}

Para el concepto de innovación, se optó por la definición de "propuesta o solución más o menos novedosa ante un problema, reto o tarea pendiente" (Christensen, 1997).

Para definir el objeto de la innovación, se partió de una premisa ortodoxa en el ámbito de la economía, que entronca con el pensamiento de Schumpeter (1975) y estandariza la Organización para la Cooperación y el Desarrollo Económicos (OECD) (Bleyen et al., 2014), según la cual, toda innovación afecta a cuatro áreas básicas: el producto, los procesos de producción y distribución, la organización y la comercialización.

En este punto se pretende romper con el planteamiento de organización periodística industrial (Anderson; Bell; Shirky, 2012). Se ha considerado que el periodismo es un proceso, más que el contenido de un medio y, por tanto, cualquier área que afecte a la creación de valor del producto o del servicio periodístico debe abordarse en este estudio. Esa premisa ha determinado el desarrollo de la matriz de análisis, recogiendo el trabajo de Amoedo et al. (2013).

Por último, el grado de una innovación viene determinado más por lo que cambia que por el factor de novedad. La academia coincide en que las innovaciones pueden ser 
radicales o marginales según el cambio que provocan en la creación de valor del producto o el servicio (Storsul; Kurmsvik, 2013).

Este trabajo se realizó en 2014, a lo largo de dos fases diferenciadas:

a) recopilación de casos y selección de la muestra;

b) análisis cualitativo y valoración final.

\section{Recopilación y selección}

Para la recopilación de los casos, se realizó una encuesta mediante correo electrónico a 20 expertos con el fin de reflejar tanto el punto de vista de la academia como el de la industria. Se consiguió recoger 15 respuestas. Se pidió que propusieran un listado de 10 iniciativas innovadoras en España. Se les sugirió mirar más allá de la categoría "medio" y se les mencionaron las citadas cuatro áreas de innovación:
a) producto y servicio;
b) procesos de producción y distribución;
c) organización;
d) comercialización.

Las respuestas de los expertos se agruparon en un listado de 60 iniciativas periodísticas innovadoras. Estas propuestas fueron cribadas para obtener una muestra definitiva de 25 casos relevantes desde el punto de vista profesional, pertinentes desde el punto de vista académico y conocidos desde el punto de vista social. Para realizar la criba, se diseñó un filtro con tres apartados: relevancia profesional, relevancia experta y relevancia social.

A cada uno de estas variables se le otorgó un 33\% de la valoración global.

Tabla 1. Síntesis del formulario para la recogida de iniciativas innovadoras

\begin{tabular}{|c|c|c|}
\hline \multirow{9}{*}{$\begin{array}{l}0 . \text { Datos básicos de la } \\
\text { iniciativa }\end{array}$} & \multirow{3}{*}{$\begin{array}{l}\text { Base tecnológica o no tecnlógica } \\
\text { Tecnológica ajena } \\
\text { No tecnológica }\end{array}$} & Tecnológica propia \\
\hline & & Tecnológica ajena \\
\hline & & No tecnológica \\
\hline & \multirow{2}{*}{$\begin{array}{l}\text { Grado de la innovación } \\
\text { Incremental (mejora sustancial) }\end{array}$} & Radical (primera vez) \\
\hline & & Incremental (mejora sustancial) \\
\hline & \multirow{4}{*}{$\begin{array}{l}\text { Objeto/Área en la que tiene lugar la innovación } \\
\text { Procesos de producción y/o distribución } \\
\text { Organización y estructura empresarial } \\
\text { Comercialización y promoción }\end{array}$} & Producto/Servicio \\
\hline & & Procesos de producción y/o distribución \\
\hline & & Organización y estructura empresarial \\
\hline & & Comercialización y promoción \\
\hline \multirow{8}{*}{ 1. Producto/Servicio } & \multicolumn{2}{|l|}{ Temas y enfoques } \\
\hline & \multicolumn{2}{|l|}{ Géneros periodísticos } \\
\hline & \multicolumn{2}{|l|}{ Multimedialidad } \\
\hline & \multicolumn{2}{|l|}{ Hipertextualidad } \\
\hline & \multicolumn{2}{|l|}{ Organización y disposición de los contenidos } \\
\hline & \multicolumn{2}{|l|}{ Contenidos creados para diferentes dispositivos } \\
\hline & \multicolumn{2}{|l|}{ Aprovechamiento del contenido generado por el usuario } \\
\hline & \multicolumn{2}{|l|}{ Difusión en varios idiomas } \\
\hline \multirow{5}{*}{$\begin{array}{l}\text { 2. Procesos de producción } \\
\text { y/o distribución }\end{array}$} & \multirow{3}{*}{ Producción } & Forma de producir los contenidos \\
\hline & & Tratamiento de la información \\
\hline & & Relaciones con la audiencia \\
\hline & \multirow{2}{*}{ Distribución } & Diversificación de los canales \\
\hline & & Tipos de registros de acceso al contenido \\
\hline \multirow{2}{*}{$\begin{array}{l}\text { 3. Organización y estructura } \\
\text { empresarial }\end{array}$} & $\begin{array}{l}\text { Estructura de la empresa, organigrama y proceso de toma de deci- } \\
\text { siones }\end{array}$ & \\
\hline & \multicolumn{2}{|c|}{ Otras: localización de la redacción, rutinas de trabajo, acuerdos y alianzas, gestión de grupos... } \\
\hline \multirow{7}{*}{$\begin{array}{l}\text { 4. Comercialización y } \\
\text { promoción }\end{array}$} & \multirow{3}{*}{$\begin{array}{l}\text { Tipo de comercialización } \\
\text { Venta directa } \\
\text { Venta indirecta }\end{array}$} & Publicitaria \\
\hline & & Venta directa \\
\hline & & Venta indirecta \\
\hline & Estrategia de imagen de marca & \\
\hline & Diseño y/o presentación del producto o servicio & \\
\hline & Acciones propias de marketing online & \\
\hline & Acciones relacionadas con la comunicación externa & \\
\hline
\end{tabular}


a) Con el fin de discriminar la relevancia profesional, se diseñó un motor de búsqueda de Google, alimentado con las principales fuentes del sector, blogs y sitios de referencia. Mediante este motor, se registró el número de items arrojado en una búsqueda con palabras clave y operadores boleanos $^{2}$. Los términos clave consistieron en variaciones sobre los nombres de la iniciativa, de la empresa y de sus promotores, y sobre su dirección web (url). Con este método se obtuvo un número aproximado de resultados por búsqueda para cada iniciativa que, posteriormente, fueron contabilizados como indicio de su relevancia profesional.

b) La relevancia experta se calculó mediante la asignación de una valoración numérica a los votos emitidos por los expertos consultados para elaborar la muestra inicial.

c) Para medir la relevancia social de cada iniciativa periodística (Napoli, 2014), se registraron datos sobre la audiencia y sobre las redes sociales. En primer lugar, se buscó el puesto que el sitio web de cada iniciativa ocupaba en el ranking nacional de $A_{l} x^{3} a^{3}$ en una semana de referencia (primera semana de septiembre de 2014). Después se contabilizó el número de seguidores en Twitter y el número de fans de cada web en Facebook, en el mismo período. Para evitar la sobreexposición de los sitios más populares, se valoró que estas iniciativas tuvieran una relación activa con sus seguidores en redes sociales. Para ello, se midió el ratio de retuits por el total de tuits en Twitter publicados (Twitonomy) y el ratio de engagement en Facebook (Likealyzer), que indica el promedio de comentarios o post compartidos.

http://www.twitonomy.com

http://likealyzer.com

A cada uno de estos registros se le asignó una ponderación específica mediante la siguiente fórmula: posición en Alexa España (20\%) + seguidores en Twitter (15\%) + ratio RTs (30\%) + fans Facebook (10\%) + ratio engagement Facebook (25\%). Aunque también cabría un reparto de porcentajes diferente e incluso la aportación de otros registros de interés.

El estudio aborda cualquier área que afecte a la creación de valor del producto o del servicio periodístico

\section{Análisis cualitativo}

La segunda parte de la metodología consistió en un análisis cualitativo de cada una de las iniciativas, teniendo en cuenta las cuatro áreas clave del proceso periodístico innovador citadas anteriormente.

Para registrar las innovaciones (tabla 1), se diseñó un formulario de Google con dos apartados:

a) en el primero se registra cada innovación por separado, se valora el grado de ruptura (radical o incremental, según se explicará posteriormente), si tienen o no base tecnológica (propia o ajena), el objeto de la innovación (las cuatro áreas mencionadas arriba) y un breve descriptor de la solución aportada para facilitar el análisis cualitativo;

b) en el segundo apartado, una vez terminada la explora-

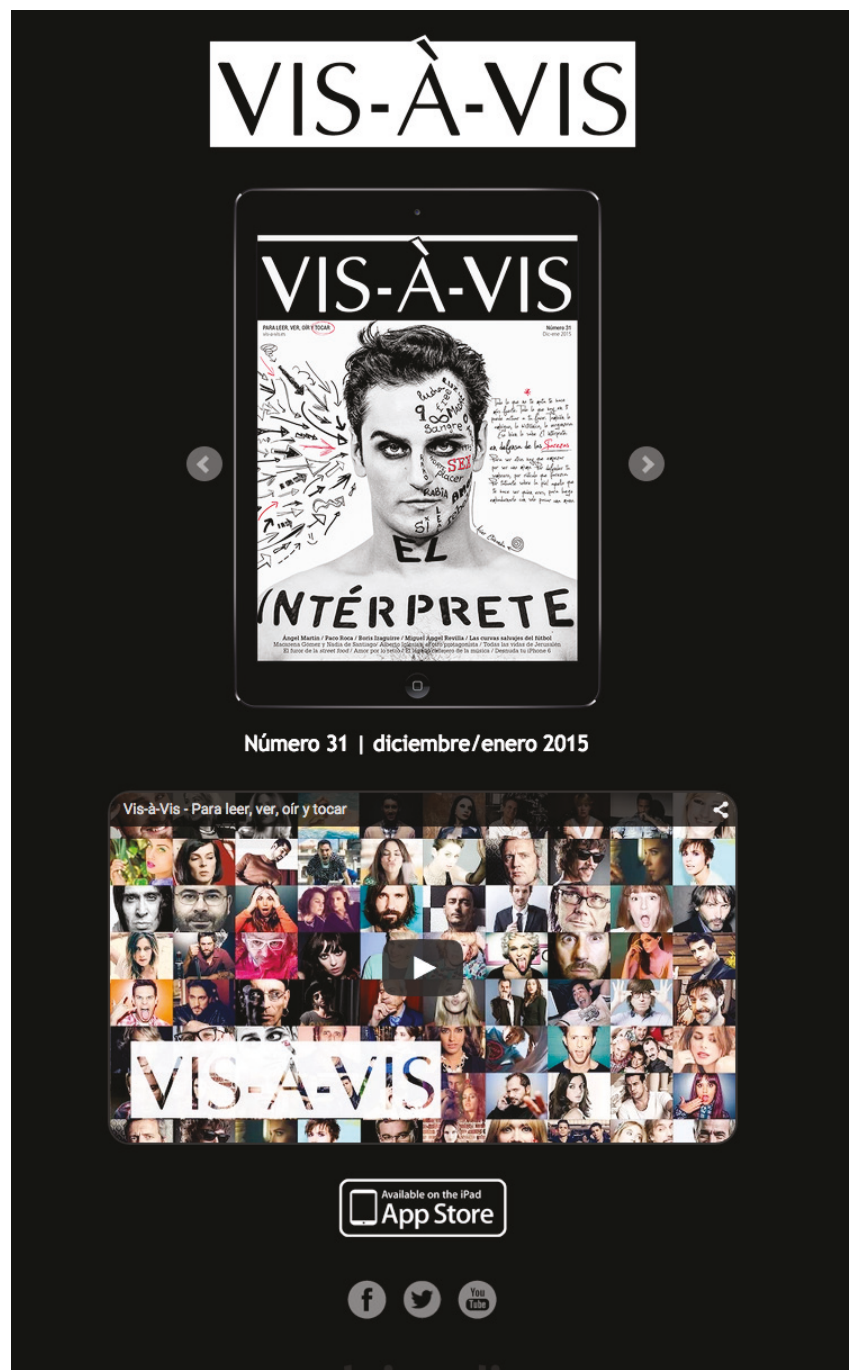

http://www.vis-a-vis.es

ción, se recogió información general sobre el caso.

La exploración del caso se realizó mediante la guía del formulario que, como se aprecia en la tabla 1, es detallada, pero no cerrada, dada la naturaleza del concepto.

Mediante esta exploración, inicialmente se registraron 625 innovaciones entre los 25 casos seleccionados. Posteriormente, se sometieron a una revisión por pares para pulir redundancias, faltas de consistencia o aspectos sin suficiente justificación. Fruto de esa discriminación, el resultado final de innovaciones se redujo a 196.

La última parte del trabajo, al final de esta segunda fase, consistió en ponderar numéricamente cada una de las innovaciones con el fin de obtener un criterio objetivable. Se optó por otorgar la misma relevancia a las cuatro áreas de la innovación, en coherencia con el punto de partida, es decir, la solución original aportada a una tarea pendiente que afecta a la creación de valor del proceso periodístico. Sin embargo, no todas las innovaciones cambian el escenario del mismo modo y no todos los agentes de un sector cambian al mismo tiempo.

Así, se optó por ponderar:

- las innovaciones de grado incremental, que suponen una adaptación o mejora leve en el contexto temporal y geo- 
gráfico establecido, con 1 punto;

- las de grado radical, aquellas aplicadas por primera vez o que implican una mejora sustancial, con 3 puntos.

Estos dos tipos de innovaciones debían haberse implementado entre julio de 2013 y julio de 2014, dentro del marco temporal del ranking. Para compensar aquellos casos que fueron primeros en innovar entre 2011 y julio 2013, se creó la categoría "grado radical antiguo" con un valor de 2 puntos. La suma final del valor de cada innovación por cada caso constituye el orden definitivo de los 25 seleccionados. Sin duda, este último criterio es el más discutible, en tanto que pueden establecerse otras ponderaciones más o menos próximas a lo aquí propuesto.

La Fundación Civio, El confidencial y la revista Vis-à-vis, a la cabeza del Ranking de innovación periodística en España

\section{Resultados}

\section{Ranking general de innovación}

La tabla 2, que recoge la clasificación general, sitúa como las iniciativas periodísticas más innovadoras a la Fundación Civio (con 22 puntos), El confidencial (19), Vis-à-vis (19),
Acuerdo (18) y eldiario.es (18). Esta clasificación pone de manifiesto que la innovación tiene lugar en los márgenes de la industria informativa tradicional. Sobre todo, si se tiene en cuenta que se trata de iniciativas como:

- Civio, una fundación sin ánimo de lucro que realiza proyectos especializados;

- Vis-à-vis, una revista interactiva para iPad;

- Acuerdo, una iniciativa que apuesta por cuestiones como la ludificación o el cómic interactivo para la evolución del reportaje.

Esta tendencia se traslada a los cinco siguientes puestos, donde se encuentran:

-Lab RTVE (17 puntos) de la televisión pública;

-Vizzuality (16 puntos), una startup enfocada al diseño y la visualización de datos y alejada del concepto clásico del periodismo;

-Infolibre (15 puntos), un nativo digital freemium; -Mongolia (13 puntos), una revista satírico-política; -Revista Don (12 puntos), un magazine para tabletas.

La difusión en papel y la innovación en la organización o la imagen de marca caracteriza a tres de las iniciativas innovadoras: La marea (11 puntos), Jot Down (10) y Panenka (10). Naukas (11) logra innovar en la divulgación científica y The objective (11), en géneros como la fotonoticia. Yorokobu (8), Porcentual (7), Qué hacen los diputados (7), Politikon (6) y SportYou (6), aunque con unos resultados más modestos, constatan la capacidad innovadora de las iniciativas de nicho.

Tabla 2. Ranking general de innovaciones periodísticas

\begin{tabular}{|c|c|c|c|}
\hline Puesto & Nombre & Web & Puntos \\
\hline 1 & Civio & http://www.civio.es & 22 \\
\hline 2 & El confidencial & http://www.elconfidencial.co & 19 \\
\hline 2 & Vis-à-vis & http://www.vis-a-vis.es & 19 \\
\hline 4 & Acuerdo & http://www.acuerdo.us & 18 \\
\hline 4 & eldiario.es & http://www.eldiario.es & 18 \\
\hline 6 & LabRTVE & http://lab.rtve.es & 17 \\
\hline 7 & Vizzuality & http://www.vizzuality.com & 16 \\
\hline 8 & Infolibre & http://www.infolibre.es & 15 \\
\hline 9 & Mongolia & http://www.revistamongolia.com & 13 \\
\hline 10 & Revista Don & http://www.revistadon.com & 12 \\
\hline 11 & Lamarea & http://www.lamarea.com & 11 \\
\hline 11 & Naukas & http://www.naukas.com & 11 \\
\hline 11 & The objective & http://www.theobjective.com & 11 \\
\hline 14 & Jot Down & http://www.jotdown.es & 10 \\
\hline 14 & Panenka & http://www.panenka.org & 10 \\
\hline 16 & Yorokobu & http://www.yorokobu.es & 8 \\
\hline 17 & Porcentual & http://www.porcentual.es & 7 \\
\hline 17 & Qué hacen los diputados & http://www.quehacenlosdiputados.net & 7 \\
\hline 19 & Politikon & http://www.politikon.es & 6 \\
\hline 19 & SportYou & http://www.sportyou.es & 6 \\
\hline 21 & Ara & http://www.ara.cat & 5 \\
\hline 21 & El extrarradio & http://www.elextrarradio.com & 5 \\
\hline 23 & Granada iMedia & http://www.granadaimedia.com & 4 \\
\hline 23 & La información & http://www.lainformacion.com & 4 \\
\hline 25 & Materia & http://www.elpais.com/elpais/ciencia.html & 3 \\
\hline
\end{tabular}

La parte final del ranking recoge proyectos minoritarios, por cuestiones geográficas: Ara y Granada iMedia; temáticas: Materia; o de soporte: El extrarradio, pero que consiguen introducir innovaciones en la participación o el periodismo de proximidad, o que se convirtieron en uno de los primeros proyectos innovadores de la red-La información.

\section{Grado de la innovación}

Civio y El confidencial se mantienen en el primer y segundo puesto en el número de innovaciones (con 15) (tabla 3), y el Lab RTVE y Vizzuality (14) ascienden hasta la tercera y cuarta posición. En la parte baja, destaca el caso de Porcentual (3), que desciende desde la decimoséptima hasta la vigesimotercera posición. Estos cambios se producen, principalmente, por el efecto del grado de las innovaciones.

Vizzuality constituye el medio con más iniciativas incrementales (13), por su capacidad para visualizar la información o transmitir su proceso creativo. Este medio sobrepasa a El confidencial (12), Civio (11) y el Lab RTVE (11). Porcentual y La información, por el contrario, presentan las cifras más reducidas en esta categoría. 
La información (2), en cambio, se sitúa en la parte alta en el número de innovaciones radicales antiguas, empatado con El confidencial (2) y Naukas (2). En cualquier caso, aquí destacan los casos de Vis-à-vis (5), el Lab RTVE (3), Infolibre (5) y La marea (4). Los dos primeros, gracias a su apuesta por los avances tecnológicos; los dos últimos, gracias a su modelo periodístico o empresarial.

Eldiario.es presenta el mayor número de innovaciones radicales (5), en especial por el carácter disruptivo de su sistema de socios. Inmediatamente después, se sitúan Civio (3), Acuerdo (3) y Mongolia (3), con innovaciones radicales en la especialización, la interactividad o la organización de eventos. Este hecho explica la elevada posición de estos dos últimos en la tabla principal, pese a no haber implantado un elevado número de innovaciones (12 y 7 respectivamente).

En términos generales, el predominio de innovaciones incrementales (141) sobre las radicales (26) refleja que la mayor parte de las mejoras introducidas en los medios españoles constituyen leves avances. El número de radicales antiguas (29), por su parte, demuestra que las innovaciones de mayor calado se realizaron antes de julio de 2014. Este hecho pone de manifiesto que la innovación no se trata de un fenómeno nuevo en la industria mediática española.

\section{Objeto de la innovación}

Los medios que destacan por sus innovaciones en cuanto al producto o servicio son, como se aprecia en la tabla 3, Acuerdo (11 puntos), la revista Vis-à-vis y el Lab RTVE (ambos con 10 puntos). En los últimos puestos se hallan eldiario.es y La marea. En el caso concreto de Acuerdo, algunas de las innovaciones en este campo tienen que ver con:

- la presentación de los contenidos mediante grandes formatos a pantalla completa;

- el predominio de la hipertextualidad;

- la organización de los menús -que rompe con los esquemas habituales-;

- la explotación de recursos visuales.

En el plano de la distribución y la producción, los medios que en-

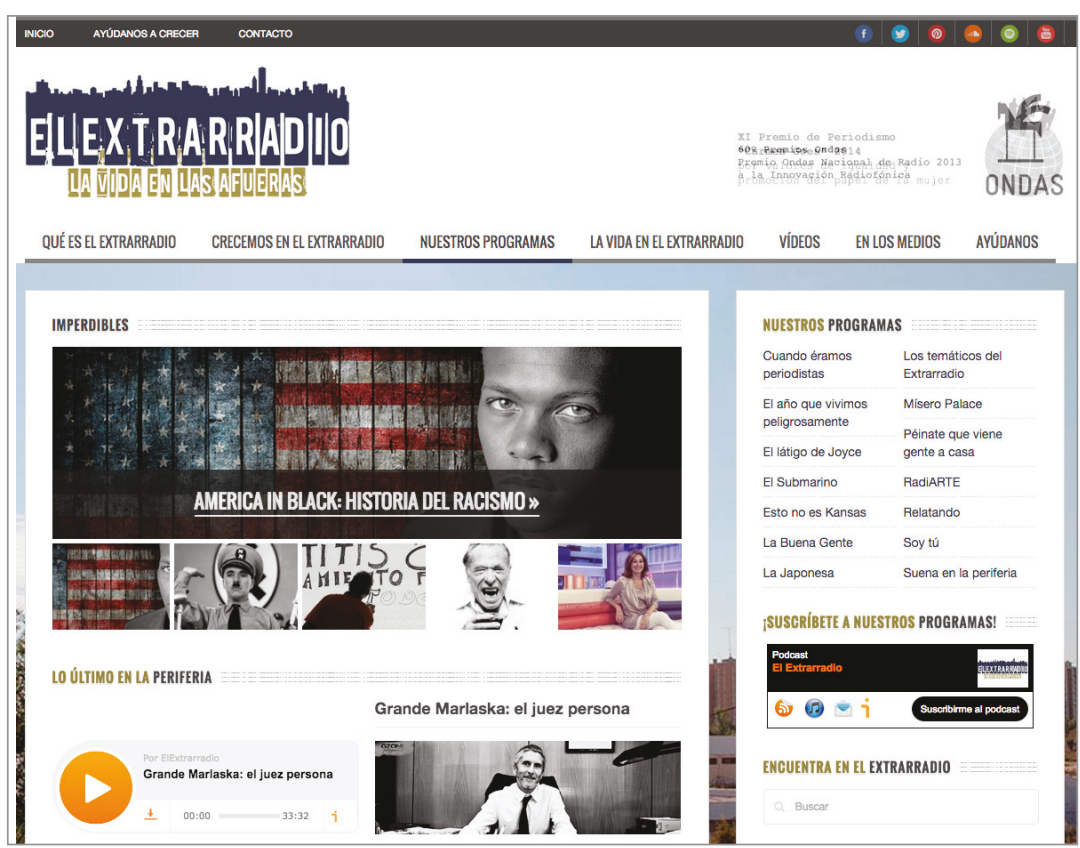

http://www.elextrarradio.com
Tabla 3. Ranking de innovaciones periodísticas según el grado de la innovación

\begin{tabular}{|c|c|c|c|c|c|c|}
\hline Puesto & Nombre & Incremental & Radical & $\begin{array}{l}\text { Radicales } \\
\text { Antiguas }\end{array}$ & Innovaciones & Puntos \\
\hline 1 & Civio & 11 & 3 & 1 & 15 & 22 \\
\hline 2 & El confidencial & 12 & 1 & 2 & 15 & 19 \\
\hline 2 & Vis-à-vis & 3 & 2 & 5 & 10 & 19 \\
\hline 4 & Acuerdo & 9 & 3 & 0 & 12 & 18 \\
\hline 4 & eldiario.es & 3 & 5 & 0 & 8 & 18 \\
\hline 6 & Lab RTVE & 11 & 0 & 3 & 14 & 17 \\
\hline 7 & Vizzuality & 13 & 1 & 0 & 14 & 16 \\
\hline 8 & Infolibre & 5 & 0 & 5 & 10 & 15 \\
\hline 9 & Mongolia & 4 & 3 & 0 & 7 & 13 \\
\hline 10 & Revista Don & 6 & 2 & 0 & 8 & 12 \\
\hline 11 & Lamarea & 3 & 0 & 4 & 7 & 11 \\
\hline 11 & Naukas & 4 & 1 & 2 & 7 & 11 \\
\hline 11 & The objective & 9 & 0 & 1 & 10 & 11 \\
\hline 14 & Jot Down & 8 & 0 & 1 & 9 & 10 \\
\hline 14 & Panenka & 5 & 1 & 1 & 7 & 10 \\
\hline 16 & Yorokobu & 6 & 0 & 1 & 7 & 8 \\
\hline 17 & Porcentual & 1 & 2 & 0 & 3 & 7 \\
\hline 17 & Qué hacen los diputados & 5 & 0 & 1 & 6 & 7 \\
\hline 19 & Politikon & 3 & 1 & 0 & 4 & 6 \\
\hline 19 & SportYou & 3 & 1 & 0 & 4 & 6 \\
\hline 21 & Ara & 5 & 0 & 0 & 5 & 5 \\
\hline 21 & El extrarradio & 5 & 0 & 0 & 5 & 5 \\
\hline 23 & Granada iMedia & 4 & 0 & 0 & 4 & 4 \\
\hline 23 & La información & 0 & 0 & 2 & 2 & 4 \\
\hline 25 & Materia & 3 & 0 & 0 & 3 & 3 \\
\hline
\end{tabular}




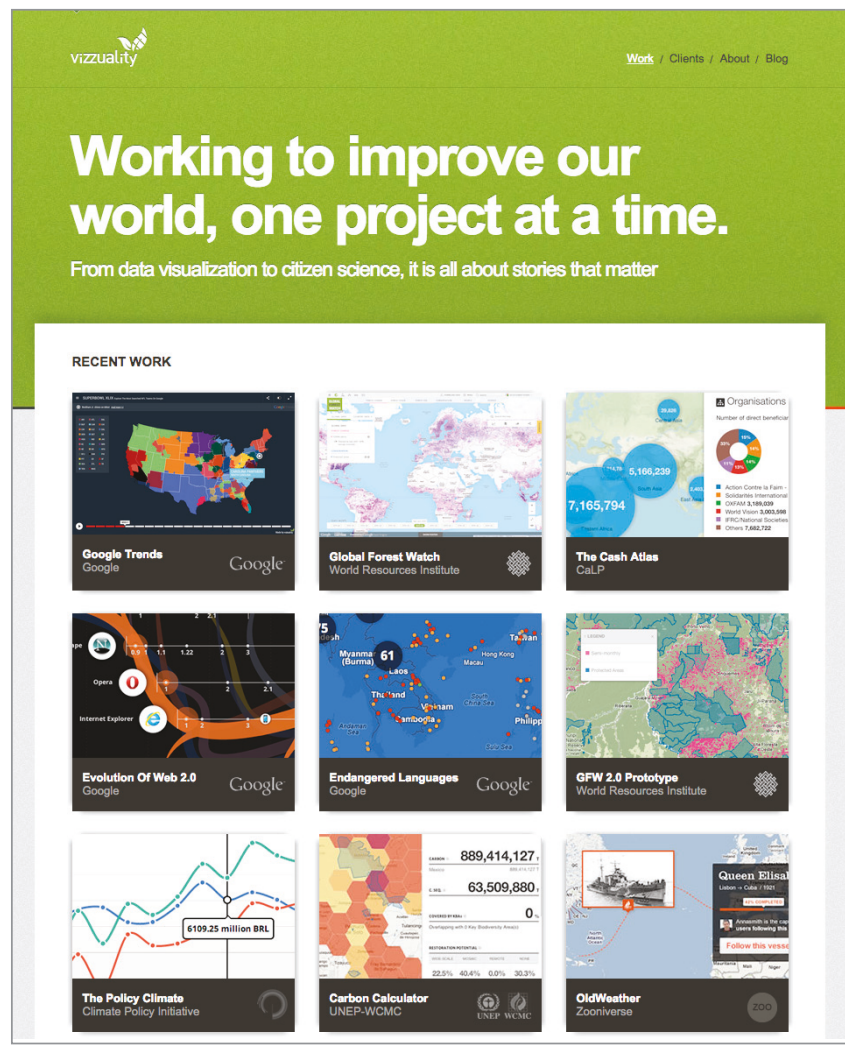

Materia. Respecto al eldiario.es, cabe destacar su especial cuidado hacia los socios, por ejemplo al primar sus comentarios respecto a los de los usuarios estándar.

Tanto en la Fundación Civio como en el caso de El confidencial sobresale la elaboración de bases de datos especializadas:

- Civio: esta característica se percibe en proyectos como España en llamas, donde se califican las sentencias, los tipos de jurados o los delitos imputables;

- El confidencial cuenta con una sección exclusiva de cotizaciones, en la que se incluye un completo listado de empresas.

El estudio aborda cualquier área que afecte a la creación de valor del producto o del servicio periodístico

En el área de la organización, encabezan el ranking dos medios impresos: La marea (5) y Mongolia (3). Más de la mitad de los medios analizados no muestra ninguna característica innovadora en esta área. La Marea fundamenta su modelo de negocio en una cooperativa donde los lectores socios pueden participar en la toma de decisiones del medio. Por su parte, Mongolia apuesta por un sólido armazón jurídico

http://www.vizzuality.com sobre el que pivota todo el proyecto.

Tabla 4. Ranking de innovaciones periodísticas según el objeto de la innovación

\begin{tabular}{|c|c|c|c|c|c|c|c|}
\hline Puesto & Nombre & Producto & Producción & Organización & Comercialización & Innovaciones & Puntos \\
\hline 1 & Civio & 6 & 9 & 0 & 7 & 15 & 22 \\
\hline 2 & El confidencial & 7 & 7 & 0 & 5 & 15 & 19 \\
\hline 2 & Vis-à-vis & 10 & 5 & 0 & 4 & 10 & 19 \\
\hline 4 & Acuerdo & 11 & 0 & 1 & 6 & 12 & 18 \\
\hline 4 & eldiario.es & 0 & 11 & 0 & 7 & 8 & 18 \\
\hline 6 & Lab RTVE & 10 & 2 & 2 & 3 & 14 & 17 \\
\hline 7 & Vizzuality & 9 & 6 & 0 & 1 & 14 & 16 \\
\hline 8 & Infolibre & 7 & 5 & 0 & 3 & 10 & 15 \\
\hline 9 & Mongolia & 5 & 1 & 3 & 4 & 7 & 13 \\
\hline 10 & Revista Don & 6 & 3 & 0 & 3 & 8 & 12 \\
\hline 11 & Lamarea & 0 & 4 & 5 & 2 & 7 & 11 \\
\hline 11 & Naukas & 5 & 0 & 1 & 5 & 7 & 11 \\
\hline 11 & The objective & 7 & 1 & 1 & 2 & 10 & 11 \\
\hline 14 & Jot Down & 4 & 3 & 0 & 3 & 9 & 10 \\
\hline 14 & Panenka & 4 & 1 & 0 & 5 & 7 & 10 \\
\hline 16 & Yorokobu & 3 & 2 & 0 & 3 & 7 & 8 \\
\hline 17 & Porcentual & 4 & 0 & 0 & 3 & 3 & 7 \\
\hline 17 & Qué hacen los diputados & 2 & 5 & 0 & 0 & 6 & 7 \\
\hline 19 & Politikon & 2 & 3 & 0 & 1 & 4 & 6 \\
\hline 19 & SportYou & 2 & 4 & 0 & 0 & 4 & 6 \\
\hline 21 & Ara & 1 & 2 & 0 & 2 & 5 & 5 \\
\hline 21 & El extrarradio & 1 & 2 & 1 & 1 & 5 & 5 \\
\hline 23 & Granada iMedia & 2 & 1 & 0 & 1 & 4 & 4 \\
\hline 23 & La información & 4 & 0 & 0 & 0 & 2 & 4 \\
\hline 25 & Materia & 1 & 0 & 0 & 2 & 3 & 3 \\
\hline
\end{tabular}


En cuanto a la comercialización, los medios que encabezan la lista son eldiario.es y la Fundación Civio (7 puntos cada uno). Eldiario.es organiza encuentros fuera de la Red con sus usuarios para compartir ideas sobre el rumbo editorial. Por su parte, la Fundación Civio ofrece cursos de formación en transparencia a instituciones y empresas.

La mayoría de las innovaciones se registran en las áreas del producto (suman un total de 100 puntos) y en la producción (77), a la que sigue de cerca el área de la comercialización (73). El campo que menos iniciativas innovadoras recoge es la organización de las empresas y proyectos (14).

\section{Aunque la innovación no está necesa-} riamente asociada a la tecnología, encuentra en ella un importante motor de cambio

\section{Base de la innovación}

Entre las innovaciones tecnológicas (con un total de 147 puntos), priman aquellas que se fundamentan en la tecnología ajena, que consiguen 89 puntos en el ranking total, frente a los 58 de las innovaciones basadas en el software propio. Las innovaciones no tecnológicas acaparan 130 puntos. Los resultados también permiten constatar que el $55,6 \%$ de las innovaciones (un total de 109) presenta una base tecnológica, frente al $44,4 \%$ que posee una base no tecnológica (87 innovaciones).

En la tabla 5 se aprecia que el medio que sobresale por sus innovaciones tecnológicas es Vizzuality, que acumula 16 puntos (con 11 innovaciones de carácter propio y 3 ajenas). Esta startup, fundada en 2007, comercializa un programa de software libre, $\operatorname{Carto} D B$, que procesa millones de datos y permite visualizarlos en mapas. El Lab RTVE, que cuenta con secciones exclusivas de documentales interactivos y webdocs, consigue 10 puntos por las innovaciones tecnológicas propias y 4 por las ajenas.

Dos publicaciones específicas para tabletas cuentan con un amplio número de innovaciones de base tecnológica. La revista Vis-á-vis, con 9 innovaciones tecnológicas (6 propias y 3 ajenas), logra 16 puntos en las innovaciones tecnológicas y 3 en las no tecnológicas. Vis-à-vis ha introducido formatos publicitarios perfectamente integrados en el entorno del iPad. Por su parte, Revista Don, con 12 puntos en las 8 innovaciones tecnológicas registradas (1 propia y
7 ajenas) ofrece un diseño adaptado a cualquier tipo de tableta (iPad o Android).

\section{Conclusiones}

La presente investigación revela que la innovación se desarrolla en los márgenes de la industria informativa tradicional: proyectos periodísticos impulsados por fundaciones, revistas para $i P a d$, empresas tecnológicas y startups. Aunque se comprueba que resulta posible innovar con independencia del formato, la mayoría de las iniciativas del ranking hallan su razón de ser en internet o lo consideran un aliado indispensable.

En términos generales, predominan las innovaciones de carácter incremental, basadas en mejoras de otras implantadas anteriormente. Las radicales se centran en cuestiones relacionadas con el modelo de negocio, la hiper-especialización, la interactividad del producto o la organización de eventos.

Se han registrado más innovaciones de carácter tecnológico que no tecnológico, lo que indica que si bien la innovación no está necesariamente asociada a la tecnología, sí encuentra en ella un importante motor de cambio. En el caso concreto de las innovaciones tecnológicas, priman aquellas fundamentadas en tecnología ajena, lo que subraya la dificultad de elaborar aplicaciones y sistemas digitales propios. En cuanto al

Tabla 5. Ranking de innovaciones periodísticas según la base de la innovación

\begin{tabular}{|c|c|c|c|c|c|c|}
\hline Puesto & Nombre & Propia & Ajena & No tecnológica & Total & Puntos \\
\hline 1 & Civio & 4 & 2 & 16 & 15 & 22 \\
\hline 2 & El confidencial & 2 & 7 & 10 & 15 & 19 \\
\hline 2 & Vis-à-vis & 10 & 6 & 3 & 10 & 19 \\
\hline 4 & Acuerdo & 13 & 1 & 4 & 12 & 18 \\
\hline 4 & eldiario.es & 0 & 6 & 12 & 8 & 18 \\
\hline 6 & Lab RTVE & 10 & 4 & 3 & 14 & 17 \\
\hline 7 & Vizzuality & 13 & 3 & 0 & 14 & 16 \\
\hline 8 & Infolibre & 0 & 8 & 7 & 10 & 15 \\
\hline 9 & Mongolia & 0 & 1 & 12 & 7 & 13 \\
\hline 10 & Revista Don & 1 & 11 & 0 & 8 & 12 \\
\hline 11 & La marea & 0 & 2 & 9 & 7 & 11 \\
\hline 11 & Naukas & 0 & 6 & 5 & 7 & 11 \\
\hline 11 & The objective & 2 & 3 & 6 & 10 & 11 \\
\hline 14 & Jot Down & 0 & 7 & 3 & 9 & 10 \\
\hline 14 & Panenka & 0 & 2 & 8 & 7 & 10 \\
\hline 16 & Yorokobu & 0 & 5 & 3 & 7 & 8 \\
\hline 17 & Porcentual & 1 & 3 & 3 & 3 & 7 \\
\hline 17 & Qué hacen los diputados & 1 & 2 & 4 & 6 & 7 \\
\hline 19 & Politikon & 0 & 1 & 5 & 4 & 6 \\
\hline 19 & SportYou & 0 & 0 & 6 & 4 & 6 \\
\hline 21 & Ara & 1 & 0 & 4 & 5 & 5 \\
\hline 21 & El extrarradio & 0 & 3 & 2 & 5 & 5 \\
\hline 23 & Granada iMedia & 0 & 1 & 3 & 4 & 4 \\
\hline 23 & La información & 0 & 4 & 0 & 2 & 4 \\
\hline 25 & Materia & 0 & 1 & 2 & 3 & 3 \\
\hline
\end{tabular}


objeto concreto de la innovación, las iniciativas se concentran en su mayoría en el producto o servicio, la producción y la comercialización, y no tanto en la organización.

La metodología propuesta pretende contribuir al esclarecimiento del concepto de innovación en el ámbito periodístico. Como queda reflejado en el estado de la cuestión, este término aún se encuentra en un terreno un tanto difuso que dificulta el consenso a la hora de definir el concepto, establecer clasificaciones y analizar sus implicaciones prácticas.

Conviene destacar las limitaciones del análisis a la hora de evaluar determinadas parcelas como, por ejemplo, el área de la organización u otros parámetros relacionados con la mejora del producto, el desarrollo de herramientas enfocadas a la analítica web o la convergencia de las redacciones.

\section{El ranking contribuye a identificar los as- pectos más innovadores del periodismo contemporáneo español}

En estos aspectos conviene profundizar en futuras investigaciones, principalmente a través de entrevistas personalizadas que amplíen los aspectos más cualitativos del presente estudio.

Cabe señalar que algunas iniciativas que componen el ranking no son, al menos por el momento, muy conocidas, lo que refleja la complejidad para hacerse un hueco en el mercado informativo y la necesidad de que estos proyectos alternativos a la industria tradicional alcancen una mayor implantación entre los consumidores.

\section{Notas}

1. El Ranking de innovación periodística fue publicado el 4 de diciembre de 2014 en la web del Master de innovación en periodismo de la Universidad Miguel Hernández de Elche. http://mip.umh.es/ranking

2. El motor puede consultarse en este enlace: http://bit.ly/motorgoogle

3. Ésta quizá no es una herramienta muy exhaustiva para la analítica, pero sí permitía obtener unos resultados homogéneos sobre el sitio web principal de cada iniciativa.

\section{Bibliografía}

Amoedo, Avelino; Arias-Robles, Félix; Carvajal-Prieto, Miguel; Negredo, Samuel (2013). "Modelos internacionales de innovación en periodismo: una propuesta metodológica". En: XIX Congreso intl de la Sociedad Española de Periodísti$c a$, Universitat Jaume I, Castellón.

Anderson, Chris W.; Bell, Emily; Shirky, Clay (2012). Postindustrial journalism: Adapting to the present: a report. Columbia University. Tow Digital Center of Journalism.

http://towcenter.org/research/post-industrial-journalismadapting-to-the-present-2

Asociación de la Prensa de Madrid (2014). Informe anual de la profesión periodística 2014. Madrid: APM.

http://www.apmadrid.es/publicaciones/informe-anual-dela-profesion-periodistica
Baumann, Sabine (2013). "Adapting to the brave new world. Innovative organisational strategies for media companies". En: Krumsvik, Arne H.; Storsul, Tanja (eds.). Media innovations. A multidisciplinary study of change. Göteborg: Nordicom, pp. 77-92. ISBN: 9789186523657

http://www.nordicom.gu.se/en/publikationer/mediainnovations

Bleyen, Valérie-Anne; Lindmark, Sven; Ranaivoson, Heritiana; Ballon, Pieter (2014). "A typology of media innovations: Insights from an exploratory study". The journal of media innovations, v. 1, n. 1, pp. 28-51.

https://www.journals.uio.no/index.php/TJMI/article/ view/800/727

http://dx.doi.org/10.5617/jmi.v1i1.800

Boczkowski, Pablo J. (2004). Digitizing the news: Innovation in online newspapers. Cambridge, Massachusetts: The MIT Press. ISBN: 9780262524391

Bruno, Nicola; Nielsen, Rasmus-Keis (2012). Survival is success. Journalistic online startups in Western Europe. Oxford: The Reuters Institute for the Study of Journalism. ISBN: 978 1907384080 https://goo.gl/MfgJQb

Bruns, Axel (2014). "Media innovations, user innovations, societal innovations". The journal of media innovations, v. 1 , n. 1, pp. 13-27.

http://eprints.qut.edu.au/69581/1/827-3739-1-PB.pdf http://dx.doi.org/10.5617/jmi.v1i1.827

Carvajal, Miguel; García-Avilés, José-Alberto; González, José-Luis (2012). "Crowdfunding and non-profit media: The emergence of new models for public interest journalism". Journalism practice, v. 6, n. 5-6, pp. 638-647. http://dx.doi.org/10.1080/17512786.2012.667267

Casero-Ripollés, Andreu (2010). "Prensa en internet: nuevos modelos de negocio en el escenario de la convergencia". El profesional de la información, v. 19, n. 6, pp. 595-601. http://www.elprofesionaldelainformacion.com/contenidos/2010/ noviembre/05.pdf http://dx.doi.org/10.3145/epi.2010.nov05

Christensen, Clayton (1997). The innovator's dilemma: When new technologies cause great firms to fail. Boston, MA: Harvard Business School Press. ISBN: 978 0875845852

Dal-Zotto, Cinzia; Van-Kranenburg, Hans (eds.) (2008). Management and innovation in the media industry. Cheltenham, UK: Edward Elgar. ISBN: 9781847201096

Dogruel, Leyla (2013). "Opening the black box. The conceptualizing of media innovation". En: Krumsvik, Arne H.; Storsul, Tanja (eds.). Media innovations. A multidisciplinary study of change. Göteborg: Nordicom, pp. 29-44. ISBN: 978 9186523657

Dogruel, Leyla (2014). "What is so special about media innovations? A characterization of the field". The journal of media innovations, v. 1, n. 1, pp. 52-69.

https://www.journals.uio.no/index.php/TJMI/article/ viewFile/665/740

http://dx.doi.org/10.5617/jmi.v1i1.665 
Escorsa-Castells, Pere; Valls-Pasola, Jaume (2003). Tecnología e innovación en la empresa. Barcelona: Universitat Politècnica de Catalunya. ISBN: 9788483017067

Fagerberg, Jan (2004). "Innovation: A guide to the literature". En: Fagerberg, Jan; Mowery, David; Nelson, Richard (eds.) The Oxford handbook of innovation. New York: Oxford U. Press, 11-28. ISBN: 9780199264551

Fumero-Reverón, Antonio (2011). “IRC 2.0. Medios para la información, la relación y la comunicación en la web 2.0". El profesional de la información, v. 20, n. 6, pp. 605-610.

http://www.elprofesionaldelainformacion.com/contenidos/2011/ noviembre/01.pdf

http://dx.doi.org/10.3145/epi.2011.nov01

García-Avilés, José-Alberto (2012). “Innovation management in crossmedia production: Leading change in the newsroom". En: Ibrus, Indrek; Scolari, Carlos A. (Eds.). Crossmedia innovations. Texts, markets, institutions. Frankfurt: Peter Lang, pp. 259-276. ISBN: 9783631622285

García-Avilés, José-Alberto; Kaltenbrunner, Andy; Meier, Klaus (2014). "Media convergence revisited: Lessons learned on newsroom integration in Austria, Germany and Spain". Journalism practice (ahead-of-print), pp. 1-12. http://dx.doi.org/10.1080/17512786.2014.885678

García-de-Torres, Elvira (2010). “Contenido generado por el usuario: aproximación al estado de la cuestión". El profesional de la información, v. 19, n. 6, pp. 585-594.

http://www.elprofesionaldelainformacion.com/contenidos/2010/ noviembre/04.pdf

http://dx.doi.org/10.3145/epi.2010.nov.04

Guallar, Javier; Rovira, Cristòfol; Ruiz, Sara (2010). "Multimedialidad en la prensa digital. Elementos multimedia y sistemas de recuperación en los principales diarios digitales españoles". El profesional de la información, v. 19, n. 6, pp. 620-631.

http://www.elprofesionaldelainformacion.com/contenidos/2010/ noviembre/08.pdf

http://dx.doi.org/10.3145/epi.2010.nov.08

Larrondo-Ureta Ainara (2005). "Presencia del formato weblog en los cibermedios: una aproximación a sus usos y funciones". Revista latina de comunicación social, n. 60.

http://www.ull.es/publicaciones/latina/200539/arrondo. htm

López-García, Xosé (2004). “Ciberperiodismo: un camino a la innovación marcado por luces y sombras". Razón y palabra, n. 41.

http://www.www.razonypalabra.org.mx/anteriores/n41/ xlopez.html

López-García, Xosé; Pereira-Fariña, Xosé (coords.) (2010). Convergencia digital. Reconfiguración de los medios de comunicación en España. Santiago: Universidad de Santiago. ISBN: 9788498873795

Micó, Josep-Lluís; Masip, Pere; Domingo, David (2013). “To wish impossible things. Convergence as a process of diffusion of innovations in an actor-network". International communication gazette, v. 75, n. 1, pp. 118-137.

http://dx.doi.org/10.1177/1748048512461765
Napoli, Philip (2014). Measuring media impact. The Norman Lear Center.

http://www.learcenter.org/pdf/measuringmedia.pdf

Nee, Rebeca C. (2013). "Creative destruction: An exploratory study of how digitally native news non-profits are innovating online journalism practices". International journal on media management, v. 15, n. 1, pp. 3-22.

http://dx.doi.org/10.1080/14241277.2012.732153

Noguera-Vivo, José-Manuel (2010). “Panorámica de la convergencia periodística: los caminos hacia la redacción Google". El profesional de la información, v. 19, n. 6, pp. 652-657. http://www.elprofesionaldelainformacion.com/contenidos/2010/ noviembre/12.pdf http://dx.doi.org/10.3145/epi.2010.nov.12

Ponce-Aguirre, Jessica-Paola (2013). "Aportes de la innovación tecnológica al periodismo iberoamericano". Premio Gabriel García Márquez de Periodismo.

http://www.fnpi.org/premioggm2014/2013/12/aportes-dela-innovacion-tecnologica-al-periodismo-iberoamericano

Ramírez-Alujas, Álvaro V. (2011). "Sobre la aplicación y desarrollo del concepto de innovación en el sector público: estado del arte, alcances y perspectivas". Revista circunstancia, v. 9, n. 26. http://papers.ssrn.com/sol3/papers.cfm?abstract_id=1820305

Salaverría, Ramón (coord.) (2005). Cibermedios. El impacto de internet en los medios de comunicación en España. Sevilla: Comunicación Social. ISBN: 8496082334

Schumpeter, Joseph (1975). Capitalism, socialism and democracy. New York: Harper.

http://digamo.free.fr/capisoc.pdf

Steensen, Steen (2009). "What's stopping them? Towards a grounded theory of innovation in online journalism". Journalism studies, v. 10, n. 6, n. 821-836.

http://dx.doi.org/10.1080/14616700902975087

Storsul, Tanja; Krumsvik, Arne H. (2013). "What is media innovation?" En: Krumsvik, Arne H.; Storsul, Tanja (eds.). Media innovations. A multidisciplinary study of change. Göteborg: Nordicom, pp. 13-26. ISBN: 9789186523657

The New York Times (2014). The New York times innovation report.

http://www.presscouncil.org.au/uploads/52321/ufiles/The New_York_Times_Innovation_Report_-_March_2014.pdf

Van-Kerkhoven, Marco; Bakker, Piet (2014). "The hyperlocal in practice: Innovation, creativity and diversity". Digital journalism (ahead-of-print), pp. 1-14.

http://dx.doi.org/10.1080/21670811.2014.900236

Weiss, Amy S.; Domingo, David (2010). "Innovation processes in online newsrooms as actor-networks and communities of practice". New media and society, v. 12, n. 7, pp. 1156-1171.

http://dx.doi.org/10.1177/1461444809360400

Westlund, Oscar; Krumsvik, Arne H. (2014). "Perceptions of intra-organizational collaboration and media workers' interests in media innovations". The journal of media innovations, v. 1, n, 2, pp. 52-74.

http://dx.doi.org/10.5617/jmi.v1i2.858 